\title{
Hypocellular Philadelphia chromosome-positive mixed-phenotype acute leukemia successfully treated with dasatinib: A case report
}

\author{
SHIN LEE ${ }^{1}$, KEI FUJITA ${ }^{1}$, HIROTO WAKAYAMA ${ }^{1}$, YUSUKE KITO ${ }^{2}$, TAKESHI HARA ${ }^{1}$ and HISASHI TSURUMI ${ }^{1}$ \\ Departments of ${ }^{1}$ Hematology and Oncology and ${ }^{2}$ Pathology, Matsunami General Hospital, Gifu 501-6062, Japan
}

Received August 30, 2021; Accepted November 25, 2021

DOI: $10.3892 / \mathrm{mco} .2021 .2466$

\begin{abstract}
Both hypocellular leukemia and Philadelphia (Ph) chromosome-positive mixed-phenotype acute leukemia (MPAL) are rare subtypes of leukemia showing unfavorable outcomes and lacking established optimal management. $\mathrm{Ph}$-positive leukemia most often presents with hypercellularity and hypoplasia is a rare condition. The present study reports an extremely rare case of hypocellular biclonal Ph-positive MPAL, which was diagnosed by biopsy and genetic analysis of bone marrow, and successfully treated with dasatinib and steroids. Briefly, a 77-year-old man presented with pancytopenia and flow cytometry of bone marrow could not be evaluated due to hypocellularity. The patient was finally diagnosed with hypocellular Ph-positive MPAL by genetic analysis and immunostaining of bone marrow biopsy. Although blood cells recovered with methylprednisolone pulse administration alone for concurrent optic neuritis, hematopoietic function rapidly normalized with dasatinib administered after definitive diagnosis of Ph-positive leukemia. Dasatinib and oral prednisolone were continued following methylprednisolone pulse administration and the patient achieved molecular complete remission (CR) on day 140 of treatment; molecular CR was maintained thereafter without any severe adverse events. In conclusion, the combination of dasatinib and a steroid may be one of the tolerable treatment options for elderly patients with hypocellular biclonal Ph-positive MPAL. Furthermore, genetic analysis and immunostaining of bone marrow biopsy can help with the diagnosis of leukemia with hypocellular bone marrow.
\end{abstract}

\section{Introduction}

Mixed phenotype acute leukemia (MPAL) is a heterogeneous and rare subtype of acute leukemia (1-3). MPAL is characterized by immunophenotypic features of a multi-cell

Correspondence to: Dr Hisashi Tsurumi, Department of Hematology and Oncology, Matsunami General Hospital, 185-1 Dendai, Kasamatsu-cho, Hashima-gun, Gifu 501-6062, Japan E-mail: htsuru@gifu-u.ac.jp

Key words: hypocellular, Philadelphia chromosome, mixed phenotype, biclonal, dasatinib lineage, and is divided into biphenotypic and biclonal types. Approximately $25 \%$ of MPAL cases have a Philadelphia chromosome $(\mathrm{Ph})(4,5)$. MPAL commonly diagnosed by the demonstration of leukemic cells expressing markers of more than one hematopoietic lineage (3). Ph-positive MPAL is more common among elderly populations, has higher white blood cell (WBC) counts at diagnosis, and resulting a worse prognosis than Ph-negative MPAL (4-7). Treatment according to the management of Ph-positive acute lymphoid leukemia (ALL) has usually been provided, but the optimal course of treatment for Ph-positive MPAL has not yet been established (8).

Hypocellular acute leukemia is another rare variant of acute leukemia that occurs more frequently among the elderly and shows a poor prognosis (9). Various regimens have been provided for hypocellular acute leukemia, but consensus remains lacking regarding the optimal treatment (10). To the best of our knowledge, no reports have addressed hypocellular MPAL despite Ph-positive and its treatment.

We report herein the case of an elderly man with hypocellular Ph-positive biclonal-type MPAL diagnosed by immunostaining of bone marrow biopsy and genetic analysis not flow cytometry because of hypocellularity of bone marrow. Dasatinib and prednisolone were useful for achieving long-term molecular remission without compromising the quality of life.

\section{Case report}

A 77-year-old Japanese man was admitted to our hospital with malaise and sudden loss of vision in the right eye. He had been treated for hypertension for the past 25 years, but had no history of hematological abnormalities. Eastern Cooperative Oncology Group performance status was 0 . Physical examination revealed conjunctival pallor, grade II/ VI ejection systolic murmur in the left parasternal area in the second to fourth intercostal space, and petechiae on bilateral upper and lower extremities. Clinical laboratory data showed: white blood cell count (WBC), 3.4x10\%/1 (neutrophils $71.0 \%$, blasts $5.0 \%$ ); hemoglobin, $7.6 \mathrm{~g} / \mathrm{dl}$; platelet count, 35x10 $/ 1$; serum ferritin level, $1,359 \mathrm{ng} / \mathrm{ml}$; serum total protein, $6.1 \mathrm{~g} / \mathrm{dl}$; albumin, $3.8 \mathrm{~g} / \mathrm{dl}$; aspartate aminotransferase, 34 IU/1; and alanine aminotransferase, 53 IU/1. However, no other abnormalities were identified, including lactate dehydrogenase level, renal function, and markers of disseminated intravascular coagulation. Right optic neuritis 

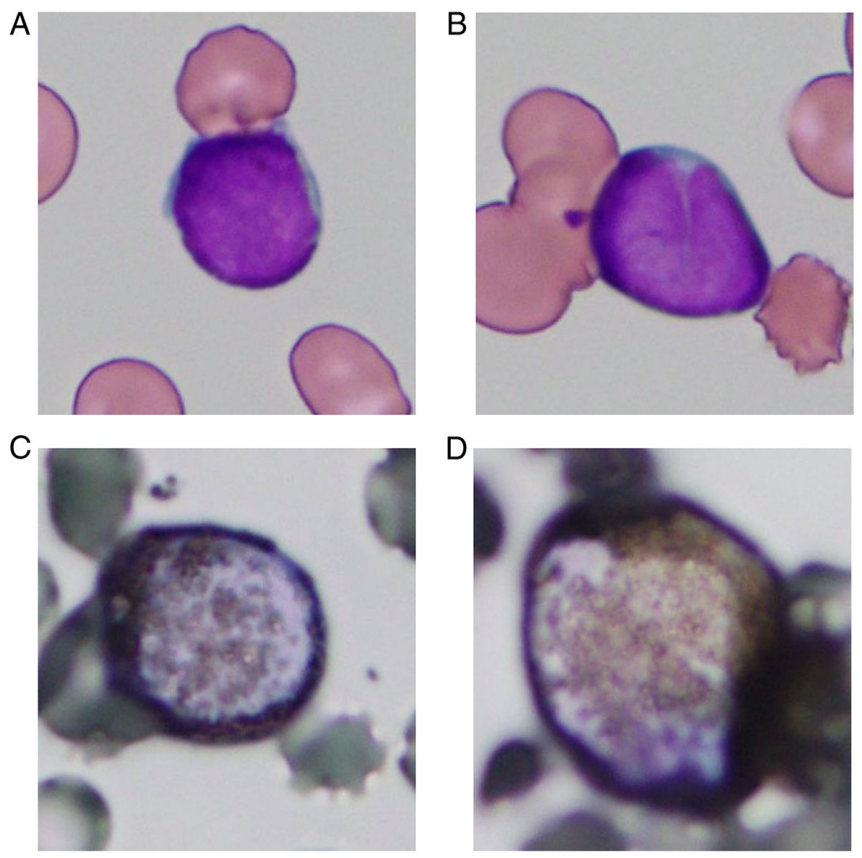

Figure 1. Blast cells in bone marrow. Bone marrow aspiration revealed hypocellular bone marrow. Blast cells on the bone marrow aspirate smear are shown. (A and B) May-Grunwald-Giemsa stain (magnification, x1,000). (C and D) Myeloperoxidase stain (magnification, x1,000).

was diagnosed and intravenous methylprednisolone pulse therapy $(1,000 \mathrm{mg} / \mathrm{body} / \mathrm{day}$, for 3 days) was immediately started. After methylprednisolone pulse therapy, oral prednisolone was continued at $25 \mathrm{mg} / \mathrm{day}$.

He was referred to us for pancytopenia. Bone marrow aspirate smears showed a very low concentration of cells (nucleated cell count, $1.0 \times 10^{4} / \mu 1$ ), myeloid/erythroid ratio, 0.5 ; and $28 \%$ blasts among non-erythroid cells. Myeloperoxidase staining of blasts yielded positive results on cytochemistry (Fig. 1). The results of flow cytometry were unevaluable due to an insufficient number of blood cells available for measurement. The level of minor BCR-ABL mRNA in bone marrow was 10,000 copies/ $\mu$ g RNA. G-banding analysis of bone marrow revealed a 46,XY karyotype. Bone marrow biopsy revealed extremely low cellularity (20\%) and proliferation of small round cells with poor nucleoli that were positive for terminal deoxynucleotidyl transferase (TdT), CD79a, and CD34, and negative for myeloperoxidase immunostaining (Fig. 2). The simultaneous presence of two different leukemic clones was demonstrated, and hypocellular Ph-positive biclonal MPAL was finally diagnosed.

Before the definitive diagnosis of leukemia, he was administered a second course of methylprednisolone pulse therapy for optic neuritis, followed by tapering of oral prednisolone to $10 \mathrm{mg} /$ day. Bone marrow examination revealed peripheral blood cells had already recovered. Twenty-eight days after admission and initial administration of steroid, dasatinib at $140 \mathrm{mg} /$ day and oral prednisolone at $30 \mathrm{mg} /$ day were started as induction therapy. After initiating induction therapy with dasatinib, peripheral blood cell count immediately recovered significantly and normalized (Fig. 3). The patient was discharged 10 days after starting induction therapy and was continuously treated with dasatinib and oral prednisolone on
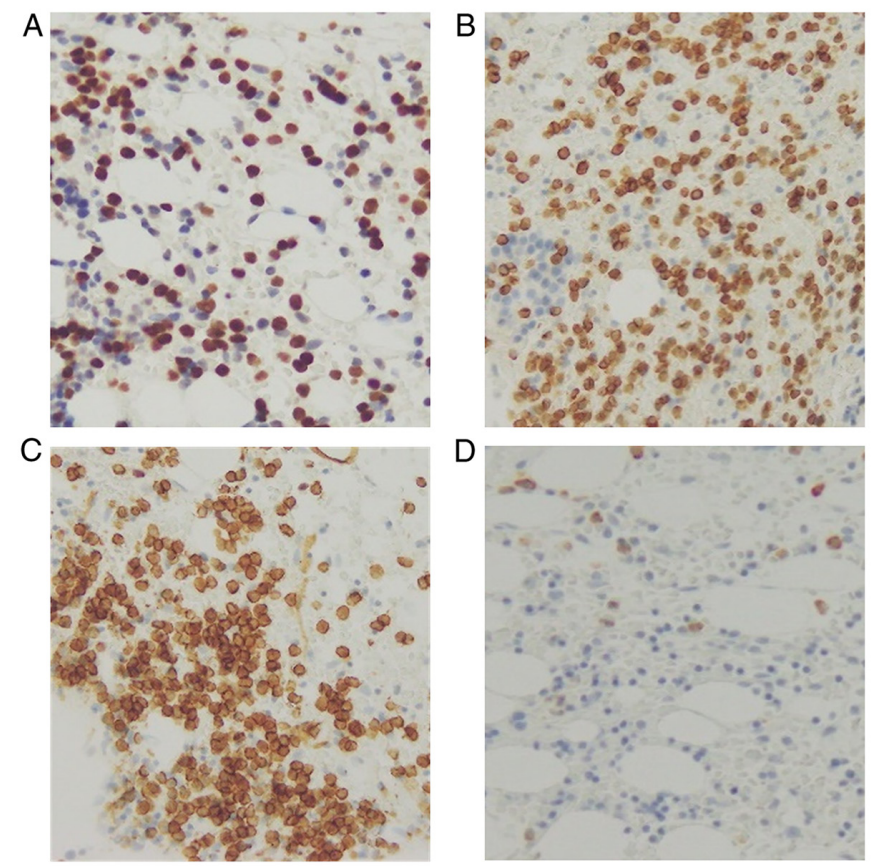

Figure 2. Immunohistochemical features of the bone marrow biopsy sample (magnification, x200). Low cellularity (20\%) and proliferation of small round cells with poor nucleoli are observed in bone marrow. These small round abnormal cells are positive for (A) terminal deoxynucleotidyl transferase, (B) CD79a and (C) CD34, and negative for (D) myeloperoxidase immunostaining.

an outpatient basis. The minor BCR-ABL fusion transcript in the bone marrow had disappeared according to real-time quantitative polymerase chain reaction at 140 days after starting induction therapy. Dasatinib was continued at $140 \mathrm{mg} / \mathrm{day}$ and oral prednisolone was tapered to $5 \mathrm{mg} / \mathrm{day}$ (Fig. 4). After achieving molecular complete remission (CR), he underwent 4 times of intrathecal chemotherapy (methotrexate $15 \mathrm{mg}$, cytarabine $40 \mathrm{mg}$, and prednisolone $10 \mathrm{mg}$ ) for central nervous system (CNS) prophylaxis. As of the time of writing, molecular $\mathrm{CR}$ has been maintained for 15 months with no serious adverse events and no loss in quality of life.

\section{Discussion}

While hypocellular leukemia has been rarely reported $(9,11)$, there have been no reports of hypocellular leukemia with $\mathrm{Ph}$-positive MPAL as in this case. It may suggest that bone marrow aspiration in a patient of hypocellular leukemia does not collect a sufficient number of cells, so the cell origin is not sufficiently evaluated, and even if it is Ph-positive MPAL, it is not properly diagnosed and underestimated. This case suggests that hypocellular leukemia found to be minor-BCR/ ABL-positive MPAL may be expected to achieve long-term survival by combination therapy with TKI and steroids, thus supporting the usefulness of genetic analysis and immunostaining of bone marrow biopsy in hypocellular leukemia.

MPAL is a rare leukemic entity that accounts for 1.6-2.8\% of adult acute leukemias $(1,2)$, and is characterized by worse prognosis than single-lineage acute leukemia. In particular, the presence of the $\mathrm{Ph}$ chromosome adversely influences the prognosis of patients with MPAL $(6,12-14)$. Although no 

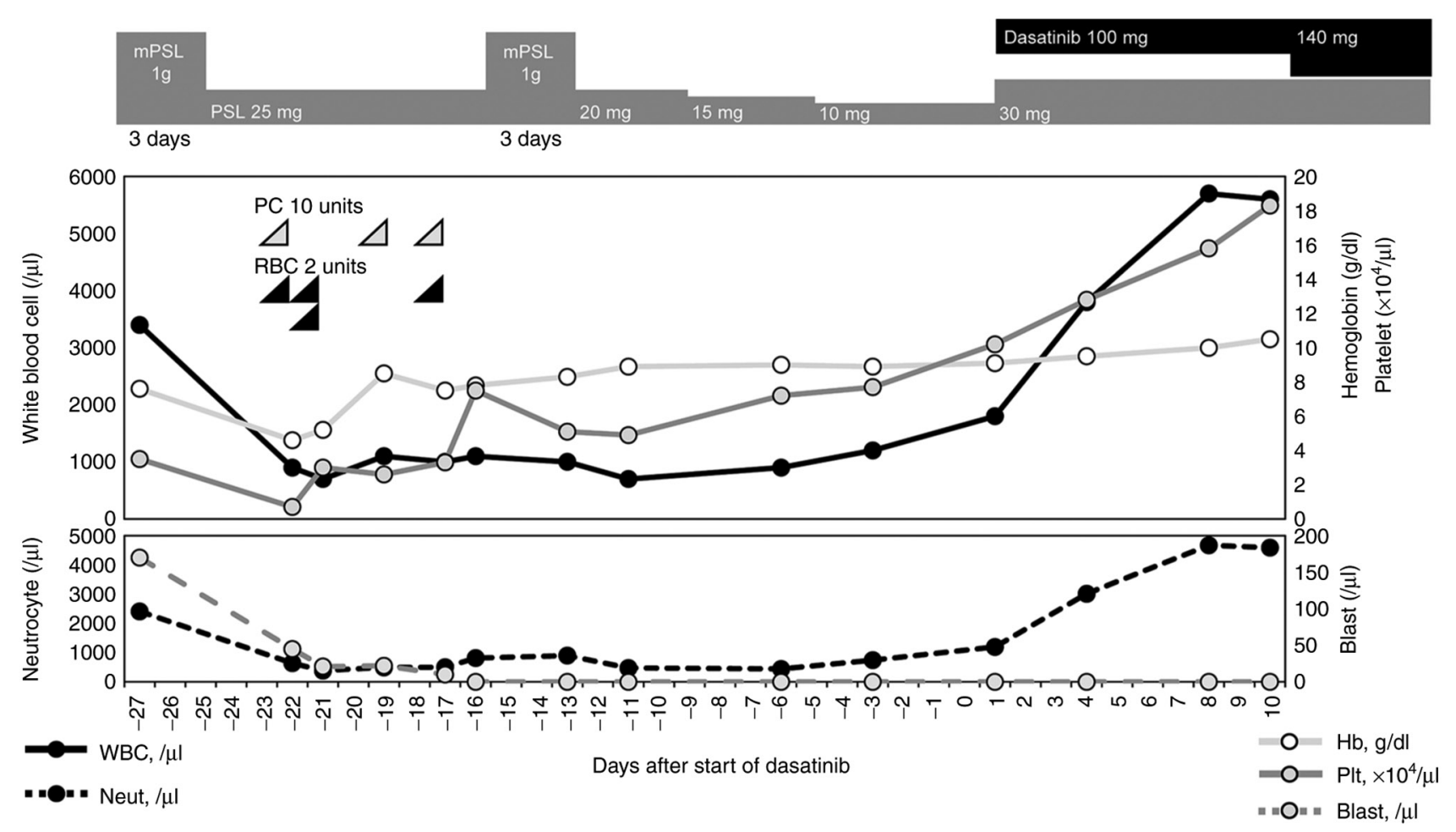

Figure 3. Clinical course during hospitalization of the present patient. Hb, hemoglobin; mPSL, methylprednisolone; Neut, neutrophils; PC, platelet transfusion; Plt, platelets; PSL, prednisolone; RBC, red blood cell transfusion; WBC, white blood cells.

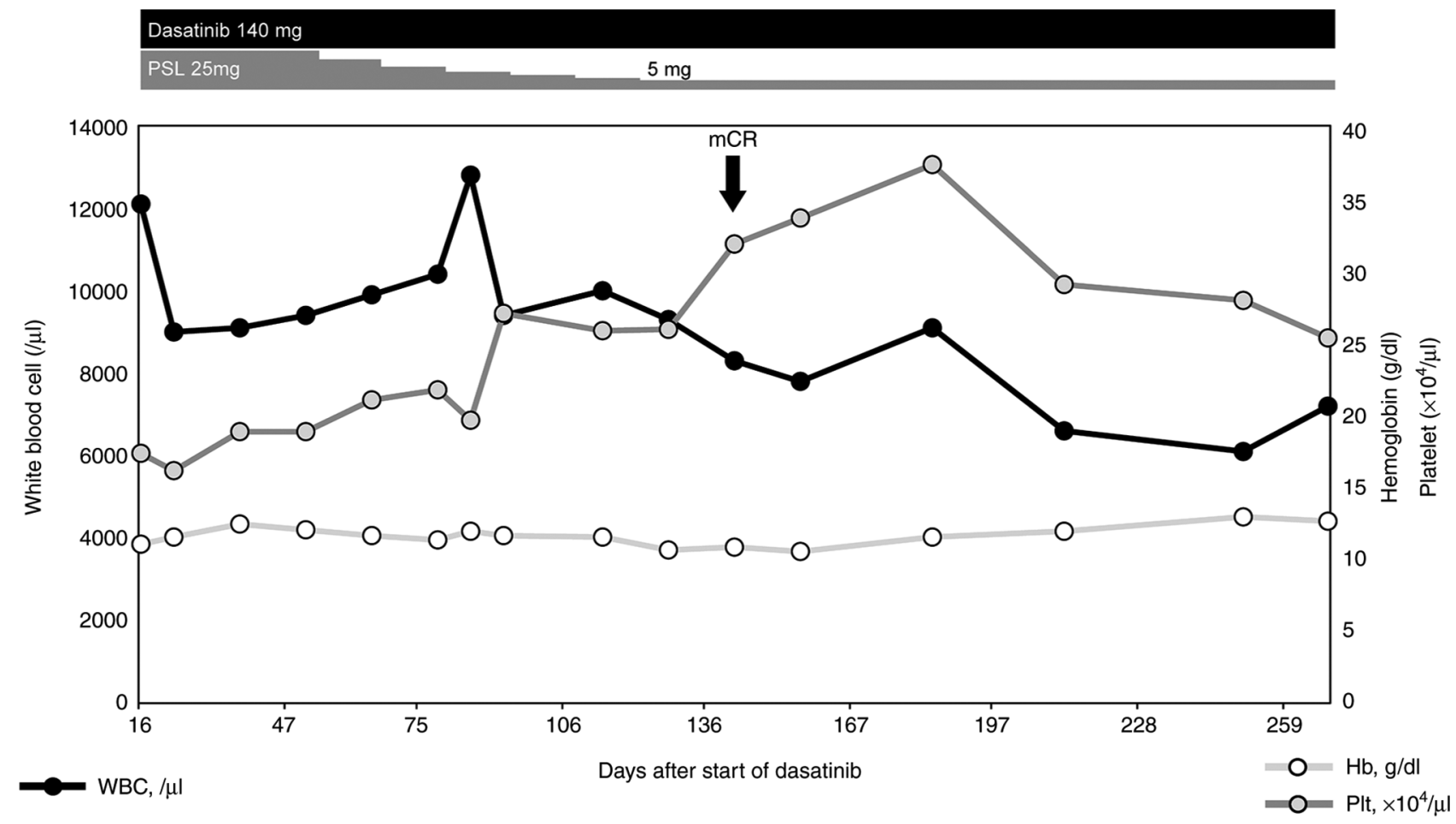

Figure 4. Clinical course of the present patient after discharge. Hb, hemoglobin; mCR, molecular complete remission; Neut, neutrophils; Plt, platelets; PSL, prednisolone; WBC, white blood cells.

consensus has been reached regarding treatment strategies for $\mathrm{Ph}$-positive MPAL, certain cases have been managed similar to Ph-positive ALL in the recent tyrosine kinase inhibitor (TKI) era, with dramatically improved outcomes $(7,8)$. Several reports have described TKI plus prednisolone or low-intensity chemotherapy as highly effective against elderly Ph-positive MPAL $(15,16)$. There have been several cases of optic neuritis as the symptom of CNS involvement by acute leukemia (17-20). Steroids have been reported to relieve optic neuritis in ALL (18). Dasatinib has also been reported to cross 
the blood-brain barrier and have an effect on the optic nerve infiltration of Ph-positive ALL $(17,21)$. The cause of the optic neuritis of the present case has not been pathologically proven, but the possibility of leukemic involvement could not be denied. Dasatinib combined with prednisolone might be effective, and the leukemic cells in the CSF may have disappeared at the time of intrathecal chemotherapy after confirmation of hematological CR. The four times of intrathecal chemotherapy also seemed to contribute to the prevention of CNS relapse and maintenance of molecular CR.

Ph-positive leukemias generally present with high WBC counts because the gene products constantly activate intracellular signal transduction pathways via markedly increased tyrosine kinase activity (22). Despite expression of the Ph chromosome, the patient in our case presented with pancytopenia and hypocellular bone marrow. $\mathrm{Ph}$-positive myelodysplastic syndrome has been reported $(23,24)$, but WBC increased after expression of the $\mathrm{Ph}$ chromosome in these cases (23), and no reports have described hypocellular bone marrow despite the presence of the $\mathrm{Ph}$ chromosome. The optimal management for hypocellular leukemia has not yet been established. The case of an elderly woman with successful control using low-dose granulocyte colony-stimulating factor and oral prednisolone has been reported (25). In our case, gradual recovery of hematopoietic function after steroid alone and rapid normalization of hematopoietic function after addition of dasatinib were observed. Early administration of steroid might have contributed to the improved hematopoietic function. In addition, since the $\mathrm{Ph}$ chromosome also inhibits the proliferation of normal blood cells (22), suppression of Ph-positive clones by dasatinib may have restored normal hematopoiesis.

We have reported an unusual case of hypocellular biclonal MPAL despite the Ph expression. Even in cases of hypocellular leukemia, physicians should keep in mind of the $\mathrm{Ph}$-chromosome expression. Genetic analysis and immunostaining of bone marrow biopsy can aid the correct diagnosis of the origin of leukemia blasts when it is unanalyzable by flow cytometry due to the hypocellular bone marrow. The clinical course of our patient demonstrated the efficacy and tolerability of dasatinib combined with steroid therapy for elderly patients with hypocellular Ph-positive MPAL.

\section{Acknowledgements}

Not applicable.

\section{Funding}

No funding was received.

\section{Availability of data and materials}

All data generated or analyzed during this study are included in this published article.

\section{Authors' contributions}

SL contributed to the conception and the design of the study, the literature review and manuscript writing, data interpretation and manuscript revision. KF and YK contributed to data interpretation, manuscript discussion, and figure creation. $\mathrm{HW}, \mathrm{TH}$ and HT helped with the design of the study, data interpretation, manuscript discussion and manuscript revision, and approved of the final manuscript version to be published. SL and KF confirm the authenticity of all the raw data. All authors read and approved the final manuscript.

\section{Ethics approval and consent to participate}

Not applicable.

\section{Patient consent for publication}

The patient provided written informed consent for publication.

\section{Competing interests}

The authors declare that they have no competing interests.

\section{References}

1. Wolach $\mathrm{O}$ and Stone RM: Optimal therapeutic strategies for mixed phenotype acute leukemia. Curr Opin Hematol 27: 95-102, 2020.

2. Weinberg OK and Arber DA: Mixed-phenotype acute leukemia: Historical overview and a new definition. Leukemia 24: 1844-1851, 2010.

3. Swerdlow SH, Campo E, Harris NL, Jaffe ES, Pileri SA, Stein $\mathrm{H}$ and Thiele $\mathbf{J}$ (eds): WHO Classification of Tumours of Haematopoietic and Lymphoid Tissues. Vol 2. Revised 4th edition. IARC Publications, Lyon, 2017.

4. Matutes E, Pickl WF, Van't Veer M, Morilla R, Swansbury J, Strobl H, Attarbaschi A, Hopfinger G, Ashley S, Bene MC, et al: Mixed-phenotype acute leukemia: Clinical and laboratory features and outcome in 100 patients defined according to the WHO 2008 classification. Blood 117: 3163-3171, 2011.

5. Weinberg OK, Seetharam M, Ren L, Alizadeh A and Arber DA: Mixed phenotype acute leukemia: A study of 61 cases using World health organization and European Group for the immunological classification of leukaemias criteria. Am J Clin Pathol 142: 803-808, 2014.

6. Weir EG, Ali Ansari-Lari M, Batista DA, Griffin CA, Fuller S, Smith BD and Borowitz MJ: Acute bilineal leukemia: A rare disease with poor outcome. Leukemia 21: 2264-2270, 2007.

7. Wang Y, Gu M, Mi Y, Qiu L, Bian S and Wang J: Clinical characteristics and outcomes of mixed phenotype acute leukemia with Philadelphia chromosome positive and/or bcr-abl positive in adult. Int J Hematol 94: 552-555, 2011.

8. Shimizu H, Yokohama A, Hatsumi N, Takada S, Handa H, Sakura T and Nojima Y: Philadelphia chromosome-positive mixed phenotype acute leukemia in the imatinib era. Eur J Haematol 93: 297-301, 2014.

9. Park HW, Lee JH, Choi SJ, Lee JH, Seol M, Lee YS, Ryu SG, Kim KH, Jang H, Seo EJ, et al: Hypoplastic acute myeloid leukemia. Blood 108: 4493, 2006.

10. Needleman SW, Burns CP, Dick FR and Armitage JO: Hypoplastic acute leukemia. Cancer 48: 1410-1414, 1981.

11. Berdeaux DH, Glasser L, Serokmann R, Moon T and Durie BG: Hypoplastic acute leukemia: Review of 70 cases with multivariate regression analysis. Hematol Oncol 4: 291-305, 1986.

12. Killick S, Matutes E, Powles RL, Hamblin M, Swansbury J, Treleaven JG, Zomas A, Atra A and Catovsky D: Outcome of biphenotypic acute leukemia. Haematologica 84: 699-706, 1999.

13. Legrand O, Perrot JY, Simonin G, Baudard M, Cadiou M, Blanc C, Ramond S, Viguié F, Marie JP and Zittoun R: Adult biphenotypic acute leukaemia: An entity with poor prognosis which is related to unfavourable cytogenetics and P-glycoprotein over-expression. Br J Haematol 100: 147-155, 1998.

14. Heesch S, Neumann M, Schwartz S, Bartram I, Schlee C, Burmeister T, Hänel M, Ganser A, Heuser M, Wendtner CM, et al: Acute leukemias of ambiguous lineage in adults: Molecular and clinical characterization. Ann Hematol 92: 747-758, 2013. 
15. Kawajiri C, Tanaka H, Hashimoto S, Takeda Y, Sakai S, Takagi T, Takeuchi M, Ohwada C, Sakaida E, Shimizu N and Nakaseko C: Successful treatment of Philadelphia chromosome-positive mixed phenotype acute leukemia by appropriate alternation of second-generation tyrosine kinase inhibitors according to BCR-ABL1 mutation status. Int J Hematol 99: 513-518, 2014.

16. Takata H, Ikebe T, Sasaki H, Miyazaki Y, Ohtsuka E, Saburi Y, Ogata $\mathrm{M}$ and Shirao K: Two elderly patients with philadelphia chromosome positive mixed phenotype acute leukemia who were successfully treated with dasatinib and prednisolone. Intern Med 55: 1177-1181, 2016.

17. Satake A, Okada M, Asada T, Fujita K, Ikegame K, Tamaki H, Fujimori Y and Ogawa H: Dasatinib is effective against optic nerve infiltration of Philadelphia chromosome-positive acute lymphoblastic leukemia. Leuk Lymphoma 51: 1920-1922, 2010.

18. Townsend JH, Dubovy SR, Pasol J and Lam BL: Transient optic perineuritis as the initial presentation of central nervous system involvement by pre-B cell lymphocytic leukemia. J Neuroophthalmol 33: 162-164, 2013.

19. Quann KA and Redner RL: The eyes have it: CNS leukemia presenting as optic neuritis. Int J Hematol 113: 311-312, 2021
20. Myers KA, Nikolic A, Romanchuk K, Weis E, Brundler MA, Lafay-Cousin L and Costello F: Optic neuropathy in the context of leukemia or lymphoma: Diagnostic approach to a neuro-oncologic emergency. Neurooncol Pract 4: 60-66, 2017.

21. Porkka K, Koskenvesa P, Lundán T, Rimpiläinen J, Mustjoki S, Smykla R, Wild R, Luo R, Arnan M, Brethon B, et al: Dasatinib crosses the blood-brain barrier and is an efficient therapy for central nervous system Philadelphia chromosome-positive leukemia. Blood 112: 1005-1012, 2008.

22. Gotoh A and Broxmeyer HE: The function of BCR/ABL and related proto-oncogenes. Curr Opin Hematol 4: 3-11, 1997.

23. Fukunaga A, Sakoda H, Iwamoto Y, Inano S, Sueki Y, Yanagida S and Arima N: Abrupt evolution of Philadelphia chromosome-positive acute myeloid leukemia in myelodysplastic syndrome. Eur J Haematol 90: 245-249, 2013.

24. Keung YK, Beaty M, Powell BL, Molnar I, Buss D and Pettenati M: Philadelphia chromosome positive myelodysplastic syndrome and acute myeloid leukemia-retrospective study and review of literature. Leuk Res 28: 579-586, 2004.

25. Islam A: Hypoplastic acute myeloid leukemia in an elderly patient. A long-term partial remission with low-dose prednisone and G-CSF. Clin Case Rep 7: 1285-1290, 2019. 